\title{
The Heart of Drug Discovery and Development: Rational Target Selection
}

\author{
Reynold Spector $^{\mathrm{a}} \quad$ Elliot S. Vesell ${ }^{\mathrm{b}}$ \\ ${ }^{a}$ Robert Wood Johnson Medical School, New Brunswick, N.J., and Harvard-MIT Program in the Health Sciences, \\ Cambridge, Mass., and ${ }^{\mathrm{b}}$ Department of Pharmacology, Pennsylvania State University College of Medicine, \\ Hershey, Pa., USA
}

\section{Key Words}

Drug target selection $\cdot$ Pathophysiology $\cdot$ Vaccine and drug discovery $\cdot$ Allopurinol $\cdot$ Statins

\begin{abstract}
Critical to the discovery and development of drugs and vaccines is the rational selection of biochemical, immunologic or molecular targets. To understand the rationale for target selection, we review strengths and weaknesses of the four main approaches: whole animal disease models; molecular targeting; epidemiology/observation studies, and genomics. After classifying diseases into those with a relatively stable pathophysiology (e.g., hypertension and gout) versus those with an unstable pathophysiology (e.g., AIDS and influenza) to aid in understanding target selection, we provide examples of successful and unsuccessful selection of drug and vaccine targets, focusing on the molecular and epidemiological/observational approaches. We discuss the reasons that molecular targeting has led to successful control of many diseases, whereas the epidemiological/observational approach has had a checkered history. We also assess the potential power of the genomic approach, specifically the curative versus controlling/preventive strategies. With combined genetic and molecular approaches and judicious use of whole animal models and properly performed epidemiology/observation studies to select the appropriate targets, the future for controlling, preventing and even curing many diseases is very bright indeed.
\end{abstract}

Copyright $\odot 2006$ S. Karger AG, Basel

\section{KARGER}

Fax +41613061234 E-Mail karger@karger.ch www.karger.com
(C) 2006 S. Karger AG, Basel 0031-7012/06/0772-0085\$23.50/0
The discovery and development of novel drugs and vaccines are difficult and expensive undertakings. In the last seven decades, many important preventive and therapeutic successes have been achieved, particularly notable among these being the elimination of various pathogeninduced diseases by antibiotic, anti-viral and anti-parasitic drugs and vaccines [1]. Critical to these discoveries has been judicious selection of drug and vaccine targets. As discussed below, notwithstanding the successes, innumerable target selection failures have occurred.

The purpose of this paper is to discuss the strengths and weaknesses of various approaches to drug target selection with a view toward the future. We propose to do that by outlining certain principles, methods and heuristic examples.

\section{Background}

In recent publications, we defined disease and illness in operational terms, discussed the philosophical and epistemological foundations of drug discovery and development, and the evidentiary requirements for drug registration with the regulatory authorities, e.g., the Food and Drug Administration (FDA) and the European Registration Authority [2-5]. We also outlined the characteristics of an ideal drug $[1,5]$. These topics are broadly relevant to the present discussion and document the consequences of successful target selection.

Elliot S. Vesell, M.D.

Department of Pharmacology, Pennsylvania State University College of Medicine 500 University Drive

Hershey, PA 17033 (USA)

Tel. +1 717531 8285, Fax +1 717531 5013, E-Mail esvl@psu.edu 
Table 1. Examples of diseases with

Stable pathophysiology
Controlling strategy
Hypertension
Osteoporosis
Gout
Benign prostatic hyperplasia
Parkinson's disease
Preventive strategy
Polio, hepatitis B, and many other viruses
Unstable pathophysiology
AIDS
Influenza
Cancer
Social diseases, e.g., homicides, accidents and war
Addictions including smoking

Table 2. Target selection approaches

Whole animal models

Molecular targeting

Epidemiology/observation studies

Genomics/proteomics

Before proceeding to target selection itself, it is worthwhile outlining an important and useful way to view many diseases from a therapeutic or preventive perspective. These diseases can be classified into two categories: those with a relatively stable pathophysiology and those with an unstable pathophysiology (table 1). In those diseases with a more stable pathophysiology, two general approaches have been successfully employed: a controlling (but not curative) approach with drugs and a preventive approach, generally with vaccines (table 1). With both approaches, the explicit objective is to control or reverse the progression of the disease or, with vaccines, prevent the disease outright. In the following examples, we will develop these concepts.

As discussed below, those diseases with a stable pathophysiology are much easier objects for either vaccine or drug target selection. For example, the three strains of polio and the hepatitis B virus do not escape our preventive vaccines. However, the unstable viruses, like Proteus, can change easily and frequently. In the examples of AIDS and influenza, caused by single-stranded RNA viruses, frequent and remarkable genomic changes, now understood at the molecular level, occur in the virus. These changes make both treatment and vaccine discovery and development difficult.

At present, there are four general approaches to drug (or vaccine) target selection (table 2). In some cases, e.g., with antibiotics and anti-tumor drugs, there are also widely employed in vitro methods. These include bacterial or cellular killing/inhibition assays in growth media or tissue culture, respectively.

\section{Whole Animal Models}

In the past, but less frequently today, whole animal models were developed to simulate human diseases. Examples of such models include simulations of arthritic disorders and more recently Huntington's disease [6]. These models were then used to screen compounds for efficacy. Although notable successes occurred, these models are expensive, cumbersome and often do not adequately mimic the human disease. However, even today, they can be useful. For example, many $\beta$-lactam antibiotics are very potent anti-bacterial agents in vitro, but in in vivo models, they work poorly or not at all. This difference can arise from high drug plasma protein binding or rapid metabolism or excretion. Such models are very useful in discarding useless clinical candidates. This approach is described by Vagelos and Galambos [7] and will not be discussed further here. Recently, transgenic models, in which mutations of single genes have been developed, have proved useful by helping to bridge the gap between the molecular and genetic approaches identified below.

\section{Molecular Targeting}

Elion and Hitchings [8], Black et al. [9], and Vagelos [7] pioneered the molecular targeting approach. Operationally, there is a three-tiered program (table 3). First, an appropriate molecular target must be selected; then a molecule identified to block the target or appropriately stimulate a defense mechanism. The examples in table 3 will be discussed. For a drug, the molecule must be absorbed, have an appropriate time of residence in the body and be safe. With vaccines, the approach is similar but instead, the body is coaxed to make antibodies or a broader response to a potential invader. With vaccines, the molecular target is generally the surface antigen(s) of the invader. With the near magic of modern medicinal chemistry and vaccine biology, a molecular or biological agent can often be found to either block/inhibit the target or stimulate body defens- 
Table 3. Molecular targeting

\begin{tabular}{lllll}
\hline Tier & Drug & & & \\
\cline { 2 - 5 } & allopurinol [8] & statins [15, 16] & $\begin{array}{l}\text { estrogen [18-20], } \\
\text { bisphosphonates, denosumab [21] }\end{array}$ & $\begin{array}{l}\text { antihypertensives } \\
{[12-14]}\end{array}$ \\
\hline Target & xanthine oxidase & HmG-CoA reductase & osteoclast function & various \\
\hline Surrogate & uric acid in blood/urine & cholesterol & $\begin{array}{l}\text { urine collagen breakdown products, } \\
\text { bone mineral density }\end{array}$ & blood pressure \\
Clinical & elimination of arthritis, & $\begin{array}{l}\text { decrease death, myo- } \\
\text { cardial infarction, stroke }\end{array}$ & $\begin{array}{l}\text { decrease fracturing, increased bone } \\
\text { strength }\end{array}$ & $\begin{array}{l}\text { decrease stroke, } \\
\text { heart attacks, heart } \\
\text { and renal failure }\end{array}$ \\
\hline
\end{tabular}

es as appropriate. Then, in animals and in clinical pharmacology studies, the effect of the drug or vaccine on the appropriate surrogate(s) can be measured. Surrogate markers of drug or vaccine activity include biochemical markers (e.g., uric acid, cholesterol, dihydrotestosterone, antibodies), physiological markers (e.g., blood pressure), and radiological markers (e.g., bone mineral density). Finally, large scale, controlled clinical trials are performed to establish the safety and clinical value of the drug or vaccine. In this process, if the correct molecular target is not chosen, the whole effort is wasted.

\section{The Example of Allopurinol and Gout [8]}

Gout is one manifestation of a disease in which excessive uric acid accumulates in the body. Excessive uric acid is causally associated not only with precipitation of uric acid crystals in the joint and its consequent, painful arthritis, but also uric acid kidney stones and, in extreme cases, crippling uric acid deposits in the cartilage and skin, tophi. Uric acid is the end-product of purine metabolism. In humans, for an unknown reason, uric acid is almost completely reabsorbed from the glomerular filtrate by a specific transport system (URAT I) in the kidney $[10,11]$. The common pathophysiology of gouty arthritis, tophi and uric acid kidney stones (aside from the cause, which can be due to acute or chronic overproduction and/or renal underexcretion of uric acid) is excessive uric acid in the blood and/or urine. Before allopurinol, medicinal chemists made drugs like probenecid to block renal reabsorption of uric acid by what we now know is the URAT I transporter $[8,10,11]$. By increasing uric acid excretion in non-stone formers, these uricosuric drugs had utility.

A new approach, blocking xanthine oxidase (the final step in uric acid synthesis in the body) with allopurinol, enabled Elion and Hitchings [8] to decrease excessive uric acid production and therefore to reduce body stores. For those with a chronic and excessive uric acid burden, the consequence of allopurinol administration was the eventual elimination of gouty arthritis, uric acid stones and tophi. Dissolution of tophi by allopurinol demonstrates the great power of (rational) molecular medicine. Moreover, the penultimate product, xanthine, which accumulates in the body when xanthine oxidase is inhibited, is soluble and nontoxic, and excreted harmlessly in the urine. In summary, the discoverers of allopurinol took advantage of the understanding of the pathophysiology of gout, uric acid kidney stones and tophi, and applied the molecular targeting approach (table 3) [8]. As long as allopurinol is taken, the disease is suppressed. Moreover, the drug 'works' in essentially everyone with the disease although higher doses may be necessary in some patients. Note, however, the disease is controlled, not cured, by allopurinol.

\section{Anti-Hypertensive Drugs and Cardiovascular Disease}

Although difficult to believe, the value of treating hypertension, regardless of cause, was only established in 1967 for those with severe hypertension [12] and in 1970 with moderate hypertension in the landmark Veterans Administration (VA) trials [13]. The notion that hypertension (blood pressure $>140 / 90 \mathrm{~mm} \mathrm{Hg}$ ) caused harm was hotly debated before these well-controlled trials [12, 13]. However, although the beneficial outcomes were clear, less strokes, renal failure and cardiac disease, the early antihypertensive drugs (e.g., those used in the VA trials such as reserpine, hydralazine and hydrochlorothiazide) had significant side effects [13].

With convincing evidence from the VA trials, the pharmaceutical industry made successively better drugs and 
rational combination products to lower blood pressure. These drugs and combination products focused on different targets: sodium excretion, the adrenergic system, calcium channels, and more recently the renin-angiotensin system. All these mechanisms (systems) maintain and in some cases elevate blood pressure. Nevertheless, hypertension is a disorder that has a relatively stable pathophysiology. Regardless of the cause, high blood pressure damages arterioles, and moderately high blood pressure causes hyaline change in arterioles all over the body. Severe high blood pressure causes actual fibrinoid necrosis [12, 13]. If blood pressure can be reduced by any safe means, i.e. by targeting any of the systems alone or in combination that maintain high blood pressure, the patient benefits. However, there do appear to be slight advantages of some of the drugs (and combinations when necessary) over others [14]. For example, the sartans appear to have the most benign side effect profile and an advantage over $\beta$-blockers in the prevention of stroke and renal dysfunction [14]. The reader is referred to other sources for further discussion [14]. However, the general point is that, in hypertension, multiple systems can each be usefully targeted either alone or in combination with clinical benefit and improved public health. Control of blood pressure with drugs constitutes a remarkable triumph of government, academic and industrial scientists. But once again this advance is not a cure.

\section{Statins and Cardiovascular Disease}

Approximately 50 years ago, the notion that elevated serum cholesterol caused harm was surmised from the fact that patients with very high serum cholesterol levels developed early and relentlessly progressive atherosclerotic cardiovascular disease. Moreover, animals fed large doses of cholesterol developed cardiovascular disease. Some epidemiologic studies, but not all, supported the notion that high cholesterol was a contributory cause of accelerated cardiovascular disease [5]. Based on these data, over several decades, attempts were made to lower cholesterol with diet, resins (which block gastrointestinal cholesterol absorption) and drugs (like MER-29 and fibrates which were either toxic or not very effective). With diet and/or these drugs, there was great controversy about the value of lowering cholesterol.

Fortunately, investigators in Japan and the United States, focusing on the enzyme HmG-CoA reductase as a molecular target, discovered and developed the statins $[15,16]$. Selection of HmG-CoA reductase as a target was rational, especially after the failure of MER-29. The latter blocked the final step in cholesterol biosynthesis, causing build-up of the toxic desmosterol, the penultimate step in cholesterol biosynthesis. HmG-CoA reductase is the ratelimiting step in cholesterol biosynthesis. Moreover, as expected, no precursor toxicity occurred in the clinical trials since the precursors are all soluble $[15,16]$. Furthermore, statins effectively lower cholesterol in humans in a gratifying dose-response fashion. The decrement in total serum cholesterol (up to 50-60\% with certain statins) allowed a real test of the 'cholesterol hypothesis' once and for all [15, 16]. In multiple, large controlled primary and secondary prevention clinical trials, statins decreased all cause mortality and also decreased myocardial infarctions, strokes and cardiovascular procedures $[15,16]$. The results were unequivocal although the exact mechanism by which statins work is unresolved [15-17]. In his evaluation of new cardiovascular drugs and procedures (e.g., angioplasty) over the last 25 years, Opie [17] placed statins first and angiotensin converting enzyme inhibitors a close second.

\section{Estrogen, Bisphosphonates and Denosumab for Osteoporosis}

Twenty years ago, based on animal work, epidemiological studies of postmenopausal women and other data, most investigators believed that estrogen loss at the time of menopause was associated with osteoclast overactivity, excessive bone reabsorption and consequent osteoporotic fractures. Bone loss was correlated with increased bone collagen breakdown products in the urine, e.g. deoxypyridinoline. Hormone replacement therapy (HRT) administered at menopause slowed osteoclast-induced bone reabsorption and, in uncontrolled trials, increased bone mineral density and appeared to reduce fractures. These data, although not well controlled, sufficed to influence the FDA to approve HRT to prevent bone loss after menopause [18]. In 2003, the Women's Health Initiative finally and unequivocally established that estrogen therapy not only increased bone mineral density but decreased fractures [19, 20], although HRT cannot be generally recommended to prevent fractures because of safety concerns [20].

Subsequently several oral bisphosphonates (e.g., alendronate, risedronate, and ibandronate) were developed and marketed [20]. These non-hormonal drugs also interfere with osteoclast function. Their molecular mechanism of action has only recently been established [20]. For the development of these drugs, collagen breakdown products in urine and blood, and bone mineral density served as surrogate markers. Effects of estrogen on these surro- 
gate markers were also employed to aid in dose selection. After proper dose selection, in large, well-controlled clinical trials, these drugs increased bone mineral density and reduced fracturing considerably (e.g., decreased vertebral fracturing by $\sim 50 \%$ in women with osteoporosis) [20]. Thus, osteoporosis and osteoporotic fracturing are no longer an inevitable consequence of aging, but a treatable and preventable condition. Moreover, essentially everyone who took these drugs had a clear physiological response.

Recently, with a clear molecular understanding of the biochemistry of osteoclast function, investigators have targeted the receptor activator of nuclear factor- $\mathrm{\kappa} B$ ligand (RANKL) for inhibition. RANKL is essential for osteoclast differentiation, activation and survival [21]. These investigators produced a human monoclonal antibody to RANKL, denosumab [21]. Given subcutaneously once every 6 months, in a dose-response fashion, denosumab increased bone mineral density comparably to the bisphosphonate alendronate given once weekly. A molecular understanding of osteoclast function allowed selection of an ideal molecular target, and discovery and development of a drug without the toxicity of bisphosphonates and hormonal therapy [21]. Denosumab exemplifies rational target selection with a biological agent, an antibody.

\section{A Cholecystokinin Antagonist - A True ‘Orphan’}

As an example of the not infrequent failure of the approach based on rational molecular target selection, we describe the discovery of a cholecystokinin (CCK) antagonist to treat various gastrointestinal disorders. In 1989, a safe, effective CCK antagonist was made [22]. CCK, a 33 amino acid hormone peptide, is released from the gut after a 'fatty' meal. Among its functions, CCK causes gallbladder contraction and emptying. As expected, the CCK antagonist, after a fatty meal, prevented the human gallbladder from contracting in a dose-response fashion [22]. Ten milligrams orally was an effective dose but no clinical use was ever identified [22]. The discovery of a CCK antagonist is an example of stunning medicinal chemistry and excellent clinical pharmacology, without a meaningful disease target. In retrospect, who would want a drug that inhibits CCK-induced gallbladder contraction?
Table 4. Hypotheses based on epidemiology/observation studies

\begin{tabular}{ll} 
Hypothesis & Result in controlled trials \\
\hline $\begin{array}{l}\text { Hormone replacement therapy } \\
\text { reduces cardiovascular events }\end{array}$ & increased events [23] \\
\hline $\begin{array}{l}\text { Megadose vitamin E reduces } \\
\text { cardiovascular events }\end{array}$ & $\begin{array}{l}\text { no effect (although } \\
\text { possibility of increase } \\
\text { not ruled out) [24] }\end{array}$ \\
$\begin{array}{l}\text { Low-fat diets reduce cardiovascular } \\
\text { events and cancer }\end{array}$ & no effect ${ }^{1}$ [25-27] \\
\hline
\end{tabular}

Calcium supplementation reduces no effect $[28,29]$

fractures and cancer

Reduction of blood homocysteine no effect [30-32]

reduces cardiovascular events

${ }^{1}$ This study alone cost USD 451 million.

\section{Epidemiology/Observation Approaches}

In recent years, a vast amount of time, money and effort have been invested in testing a series of hypotheses generated by cross-sectional, case-control (retrospective) or cohort (prospective but not randomized) epidemiology/observation studies. Based on large numbers of such positive studies, these hypotheses included: do women who use hormone replacement therapy have less cardiovascular disease than those who do not? Do women who ingest calcium supplements have fewer fractures and cancers? Does lowering plasma homocysteine with vitamins or ingestion of megadoses of vitamin E reduce cardiovascular disease? Does less dietary fat decrease cardiovascular disease and cancer? These questions, supported by multiple epidemiological/observation studies, became the basis for launching large controlled trials to prove or disprove them. Moreover, before definitive studies were done, there were widespread recommendations, based mainly on the published epidemiological/observation studies, to lower serum homocysteine, use HRT after menopause, eat a low-fat diet, and ingest megavitamin $\mathrm{E}$ and calcium supplements daily. The negative results that emerged appear in table 4 [23-32].

Why were these hypotheses and targets so erroneous? Why did the epidemiological/observation results differ so much from the randomized controlled trials, the scientific and FDA 'gold' standard (table 4) $[2-5,13]$ ? Several reasons are noted in table 5 [3,33-35]. First, in nonrandomized trials (whether retrospective case-control or prospective cohort trials), it is impossible to be certain 
Table 5. Reasons for selection of erroneous targets from epidemiological/observational studies [3, 33-35]

Bias (not randomized)

Post-hoc hypotheses

Validation of methods either inadequate or not done

Hill evidentiary criteria not followed

that there is no bias. Second, proper methodologic validation was sometimes omitted or poorly performed, or instruments of marginal utility employed $[3,33,35]$. In a key trial, the instrument to measure one of the two main variables was not validated [3]. Third, if multiple post hoc analyses of data are performed (data-dredging), some will be positive by chance alone. Finally, decades ago, Bradford Hill published criteria for assessing causality in epidemiological/observation studies; these were recently reviewed by us [2]. Hill's rigorous criteria were not followed in the epidemiology/observation studies that provided the hypotheses for the studies noted in table 4 . Hence, target selection was fraught with the likelihood of grievous error. In depth criticisms of the epidemiological/observation approach to target selection have recently been published [2, 3, 33-35].

What consequences and reverberations arise from the studies in table 4 ? First, in our opinion, the epidemiological/observation approach as currently practiced (table 4) has wasted time and resources, with the selection of many worthless targets. Had the investigators followed the scientific and evidentiary principles developed and espoused by Bradford Hill, this would have been avoided $[2,3]$. Second, the results of the controlled trials are sometimes ignored or not accepted by the proponents of the epidemiology approach, even though the controlled trial results are usually unambiguous (table 4 ). For example, both estrogen alone and in combination with progestins (in postmenopausal women) not only did not prevent but actually increased cardiovascular events [23]. This is not surprising since estrogen in younger women increases both arterial and venous clotting. When asked about the resistance to the acceptance of the results of these controlled estrogen trials by the proponents of estrogen for reducing cardiovascular risk, Elias Zerhouni, Director of the National Institutes of Health, said, 'Often in science, the reaction to a new finding is directly proportional to the strength of the dogma it overturns. People are still in denial of the theory of relativity, too' [18]. Yet, proponents of these disproved hypotheses continue to mine the studies (data-dredge) to justify their beliefs [36, 37].
Why does this happen? Reasons abound. Like Hume, we believe there are psychological explanations for some of these phenomena, especially beliefs dealing with hormones, diet and vitamins. Deep-rooted human notions exist that hormones preserve youth and vitality, that you 'are what you eat' and 'if a little (vitamin) is good for you, more is better' [24]. This non-rational thinking manifests itself in food fads and generally useless megavitamin therapy [24]. Proponents of such fads ignore the profound homeostatic mechanisms as well as inter-conversion of fats, carbohydrates and protein in the body, the wisdom of the body. Proponents also ignore the decades of work in nutrition showing that humans only require certain vitamins, essential fatty and amino acids, $30 \mathrm{~g}$ of quality protein daily, certain minerals like potassium and enough calories from any source to maintain ideal body weight. The New York Times editorial page recently disparaged and lampooned the continuously evolving food fads and erroneous epidemiological/observation studies [38]. There are also other nonscientific reasons for the continued inappropriate performance and publication of poor quality epidemiology/observation studies $[3,4,18]$.

\section{Genetic Targeting}

The sequencing of the genome and the possibility of gene therapy, or even gene repair or alteration, are exciting developments that hold promise for the future. We have little doubt that genetic targeting in the broad sense will eventually yield great advances in rational molecular targeting. It may allow an attack on the cause of many diseases rather than just their pathophysiology as outlined above.

A historical triumph in clinical genetic targeting lies in the development of finasteride for treatment of benign prostatic hyperplasia (BPH) $[7,39]$. A common disease of older men, BPH causes difficulty in urinating because of constriction of the intraprostatic urethra by prostate overgrowth. The discoverers of finasteride astutely observed that patients with deficiency of $5 \alpha$-reductase, the enzyme that converts testosterone to dihydrotestosterone (DHT), had very small prostates. Moreover, in adult males there was solid evidence that secondary sex characteristics and sexual function, with the exception of prostate size, depended on testosterone, not DHT. Hence, a specific inhibitor of $5 \alpha$-reductase was sought, discovered, developed, and shown to reduce DHT in the prostate by over $90 \%[7,39]$. Moreover, in clinical trials of $\mathrm{BPH}$ patients, the prostate shrunk by $20 \%$, and there was relief of symp- 
toms $[7,39]$. As anticipated, finasteride also decreased surgery for $\mathrm{BPH}$ and acute urinary retention by about $50 \%$ [39]. This is one of the best examples of successful molecular targeting based on careful analysis of clinical genetics and biochemistry. However, one unexpected observation was that, with maximum doses of finasteride, there was still approximately $20 \%$ as much DHT in the serum. This turned out to arise from a second $5 \alpha$-reductase present in the skin and other organs [40]. Subsequently, dutasteride, an inhibitor of both $5 \alpha$-reductases (a dual inhibitor), was discovered and developed for BPH [40].

\section{Conclusion}

Rational target selection has led to the discovery and development of many useful drugs and vaccines, especially for diseases with stable pathophysiology (table 1$)[1,5$, 13]. In many cases, standard once-a-day or less frequent dosing achieves clinical results in essentially all patients who take the drugs $[1,5]$. Many of these effective and safe drugs are, or will soon become generic, thus decreasing their cost. Even for diseases with relatively unstable pathophysiology, progress is evident. For example, in AIDS, concurrent inhibition of several molecular targets (e.g., protease and reverse transcriptase) has led to effective therapy. Successful therapy of influenza will almost certainly require concurrent use of several drugs targeted to different viral functions. Moreover, we believe, with genomics and a better understanding of causal mechanisms, many now inadequately treated diseases will eventually be cured or prevented. We can step beyond just control of pathophysiology (table 3). With combined genetic and molecular approaches and judicious use of whole animal models and properly performed epidemiology/observation studies, the future is very bright indeed.

\section{Acknowledgments}

The authors wish to thank Michiko Spector, Jennifer W. Schwing and Rita Nacchio-Wells for their aid in the research for and preparation of the manuscript.

\section{References}

1 Spector R: Progress in the search for ideal drugs. Pharmacology 2002;64:1-7.

-2 Spector R, Vesell ES: The pursuit of truth: role of epidemiology/observation studies. J Clin Pharmacol 2000;40:1205-1210.

3 Spector R, Vesell ES: Which studies of therapy merit credence? Vitamin E and estrogen as cautionary examples. J Clin Pharmacol 2002; 42:955-962.

-4 Spector R, Vesell ES: A rational approach to the selection of drugs for clinical practice. Pharmacology 2002;65:57-61.

5 Spector R, Vesell ES: The power of pharmacological sciences: the example of the proton pump inhibitors. Pharmacology 2006; 76: 148-156.

6 Rebec GV, Barton SJ, Ennis MD: Dysregulation of ascorbate release in striatum of behaving mice expressing the Huntington's disease gene. J Neurosci 2002;22:RC202.

7 Vagelos R, Galambos L: Medicine, Science and Merck. Cambridge, Cambridge University Press, 2004

8 Rundles RW, Wyngaarden JB, Hitchings GH, Elion GB: Drugs and uric acid. Ann Rev Pharmacol 1969:9:345-362.

-9 Black JW, Duncan WAM, Durant CJ, Ganellin CP, Parsons EM: Definition and antagonism of histamine $\mathrm{H}_{2}$-receptors. Nature 1972;236: 385-390.
10 Enomoto A, Kimura H, Chairoungdua A, et al: Molecular identification of a renal urateanion exchanger that regulates blood urate levels. Nature 2002;417:447-451.

-11 Ichioka K, Hosoyamada M, Hisatome I, et al: Clinical and molecular analysis of patients with renal hypouricemia in Japan - influence of URAT-1 gene on urinary urate excretion. J Am Soc Nephrol 2004;15:164-173.

12 Veterans Administration Cooperative Study Group: Effects of treatment on morbidity in hypertension. JAMA 1967;202:1028-1034.

13 Spector R: The Scientific Basis of Clinical Pharmacology: Principles and Examples. Boston, Little Brown, 1986.

14 Jackson EK: Renin and angiotension; in Brunton LL (ed): Goodman and Gilman's the Pharmacological Basis of Therapeutics. New York, McGraw-Hill, 2006, pp 789-821.

15 Randomized trial of cholesterol lowering in 4444 patients with coronary heart disease: the Scandinavian Simvistatin Survival Study (4S). Lancet 1994;344:1383-1389.

16 Baigent C, Keech A, Kearney PM, et al, Cholesterol Treatment Trialists' (CTT) Collaborators: Efficacy and safety of cholesterol-lowering treatment: prospective meta-analysis from 90,056 participants in 14 randomized trials of statins. Lancet 2005;366:1267-1278.

17 Opie LH: Controversies in cardiology. Lancet 2006;367:13-14.

18 Spake D: The menopausal marketplace. US News World Rep 2002;133:42-50.
19 Cauley JA, Robkins J, Chen Z, et al: Effects of estrogen plus progestin on risk of fracture and bone mineral density: the Women's Health Initiative randomized trial. JAMA 2003;290: 1729-1738.

20 Raisz LG: Pathogenesis of osteoporosis: concepts, conflicts and prospects. J Clin Invest 2005; 115:3318-3325.

21 McClung MR, Lewiecki EM, Cohen SB, et al, AMG 162 Bone Loss Study Group: Denosum$\mathrm{ab}$ in postmenopausal women with low bone mineral density. N Engl J Med 2006;354:821831.

22 Liddle R, Gertz BJ, Kansyama S: Effect of a novel cholecystokinin (CCK) receptor antagonist, MK-329, on gallbladder contraction and gastric emptying in humans. Implications for the physiology of CCK. J Clin Invest 1989; 84:1220-1225.

23 Anderson GL, Limacher M, Assaf AR, et al, Women's Health Initiative Steering Committee: Effects of conjugated equine estrogen in postmenopausal women with hysterectomy: the Women's Health Initiative randomized controlled trial. JAMA 2004;29:1701-1712.

24 Brown BG, Crowley J: Is there any hope for vitamin E. JAMA 2005;293:1387-1390.

25 Howard BV, Van Horn L, Hsia J, et al: Low-fat dietary pattern and risk of cardiovascular disease: the Women's Health Initiative Randomized Controlled Dietary Modification Trial. JAMA 2006;295:655-666. 
26 Beresford SAA, Johnson KL, Ritenbaugh C, et al: Low-fat dietary pattern and risk of colorectal cancer: the Women's Health Initiative Randomized Controlled Dietary Modification Trial. JAMA 2006;295:643-654.

-27 Prentice RL, Cann B, Chlebowski RW, et al: Low-fat dietary pattern and risk of invasive breast cancer: the Women's Health Initiative Randomized Controlled Dietary Modification Trial. JAMA 2006;295:629-642.

-28 Jackson RD, LaCroix AZ, Gass M, et al, Women's Health Initiative Investigators: Calcium plus vitamin D supplementation and the risk of fractures. N Engl J Med 2006;354:669683.

29 Wactawski-Wende J, Kotchen JM, Anderson GL, et al, Women's Health Initiative Investigators: Calcium plus vitamin D supplementation and the risk of colorectal cancer. N Engl J Med 2006;354:684-696.
30 Davey Smith G, Ebrahim S: Folate supplementation and cardiovascular disease. Lancet 2005;366:1679-1681.

31 Bonaa KH, Njolstad I, Ueland PM, et al: Homocysteine lowering and cardiovascular events after acute myocardial infarction. N Engl J Med 2006;354:1578-1588.

32 Heart Outcomes Prevention Evaluation (HOPE) 2 Investigators: Homocysteine lowering with folic acid and B vitamins in vascular disease. N Engl J Med 2006;354:15671577.

33 Col NF, Pauker SG: The discrepancy between observational and randomized trials of menopausal hormone therapy: did expectations shape experience? Ann Intern Med 2003;139: 923-929.

34 Ioannidis JPA: Contradicted and initially stronger effects in highly cited clinical research. JAMA 2005;294:218-228.

35 Sempos CT: Some limitations of semiquantitative food frequency questionnaires. Am J Epidemiol 1992;135:1127-1132.
36 Grodstein F, Manson JE, Stampfer M: Hormone therapy and coronary heart disease: the role of time since menopause and age at hormone initiation. J Womens Health (Larchmt) 2006;15:35-44.

37 Hsia J, Langer RD, Manson JE: Conjugated equine estrogens and coronary heart disease. Arch Intern Med 2006;166:357-365.

38 Editorial: Low-fat diets flub a test. NY Times 2006;Feb 9:A26.

39 McConnell JD, Bruskewitz R, Walsh P, et al: The effect of finasteride on the risk of acute urinary retention and the need for surgical treatment among men with benign prostatic hyperplasia. Finasteride Long-Term Efficacy and Safety Study Group. N Engl J Med 1998; 338:557-563.

40 Roehrborn CG, Marks LS, Fenter T, et al: Efficacy and safety of dutasteride in the fouryear treatment of men with benign prostatic hyperplasia. Urology 2004;63:709-715. 